\title{
La lógica manicomial. Un análisis de los imaginarios que despierta la locura en los trabajadores del hospital general ${ }^{1}$
}

\section{Asylum logic. An analysis of the imaginary that recalls the madness in the health workers of a general hospital}

\author{
Pablo Carcovich ${ }^{2}$
}

\section{Resumen}

Este trabajo se propone realizar una lectura de la práctica de internación por salud mental en un hospital general de la ciudad de Rosario entre 2010 y 2013. En aquel momento las internaciones por crisis subjetivas, si bien contaban con antecedentes y circuitos institucionalizados, adquirían un nuevo marco legal a partir de la sanción de la ley nacional de salud mental que nombra a esta institución como lugar sustituto para llevarlas adelante.

El objetivo es problematizar sobre determinados aspectos que emergen en estas prácticas, que tienen que ver con los imaginarios que despierta la locura en los trabajadores de la salud. Se parte de la hipótesis de que el hospital puede ser un escenario en el que se actualice la lógica manicomial, obstaculizando la realización de las nuevas formas de tratamiento del padecimiento psíquico, presentes en las leyes de salud mental.

El acercamiento a la problemática se realizó a partir de un análisis bibliográfico de los movimientos de desmanicomialización de diferentes países, así como el vivido en los últimos años en la provincia de Santa Fe. El tiempo de trabajo en el hospital como residente de la carrera permitió formular las preguntas necesarias en relación al modo de llevarse adelante las internaciones por crisis subjetivas y, de esa manera, realizar luego una serie de entrevistas a informantes clave (psicóloga, enfermeros, médica) y de la gestión provincial. Entre los hallazgos obtenidos se destaca que el paciente con padecimiento psíquico presenta mayores complicaciones a la hora de recibir asistencia en el hospital general, persistiendo la especificidad de la locura a la institución manicomial. Estas dificultades están asociadas al imaginario de la locura presente en los trabajadores y a una destitución del otro como semejante, y remiten a la constante dificultad para incluir los problemas de salud mental en los efectores de salud. Se destaca la necesidad de afrontar estratégicamente este problema para avanzar en el cumplimiento de la legislación vigente, contemplando que la complejidad de estas

\footnotetext{
${ }^{1}$ El presente artículo fue reescrito sobre la base del Trabajo Final Integrador de la Carrera de Especialización en Psicología Clínica, Institucional y Comunitaria (UNR): "Locura, desmanicomialización y salud mental” (Carcovich: 2015) dirigido por Claudio Cúneo.

${ }^{2}$ Psicólogo. Especialista en Psicología Clínica, Instituciona y Comunitaria. Psicólogo del Dispositivo de Salud Mental para ciudadanos detenidos o bajo medidas de seguridad. Dirección Provincial de Salud Mental. Ministerio de Salud. Provincia de Santa Fé. pablocarcovich@gmail.com
} 
prácticas, no se resuelve desde la burocratización de las mismas.

\section{Palabras clave}

locura - hospital general - lógica manicomial imaginarios - trabajadores de la salud

\section{Abstract}

This work aims to realize a reading of the practice of internment of mental health in a general hospital of Rosario's city between 2010 and 2013. At that moment, the internments that concerned subjective crises (though they were relying on precedents and institutionalized circuits), were acquiring a new legal frame due to the sanction of the national mental health law which stated that general hospitals were an alternative institution to psychiatric hospitals.

The main objective of this work is to enable a more complex analysis of certain aspects that emerge in these practices. We mainly focus in the imaginary that the madness recall in the workers of the health. The hypothesis consists in the idea that the hospital can be a scene in which the asylum logic is updated, interferring the accomplishment of the new ways of mental suffering treatment proposed by the law.

The approach to the problems was made through a bibliographical analysis of the demanicomialization processes carried out in different countries, as well as the experience of the Santa Fe Local Territory during the last years. I worked at the hospital as a resident of the Specialization Course and this allowed me to formulate several questions related to the way in which the internments for subjective crises were carried out there, and I could make several interviews to key informants (psychologist, nurses, doctor) of the Local Public Institution Management. Among the collected information, it is emphazised that the patient with psychic suffering presents major complications at the moment of receiving assistance in the general hospital, due to the persisting idea of being a special pacient for special institution (asylum). These difficulties are associated to the imaginary of madness which is still present in the workers and which, as a consequence, carries a dismissal of the other one as a similar one. This permanent difficulty hampers to include the problems of mental health in the general hospitals. It's outlined the need to deal strategically whith this problem in order to advance in the fulfillment of the legislation in force, bearing in mind the complexity of these practices. They will not be solved through a process of beaucratisation.

\section{Keywords}

Madness - general hospital - asylum logical imaginary - bealth workers

\section{La locura: formas y reformas.}

Podríamos decir, a grandes rasgos, que los movimientos de reformas del manicomio en los diferentes países han intentado modificar las formas de tratamiento del objeto locura, produciendo desde lo teórico e institucional, diferentes maneras de abordaje. Si bien estos procesos, conocidos como movimientos de desmanicomialización, han tenido diferentes alcances, una estrategia fundamental fue el pasaje del dispositivo manicomial y el encierro hacia los modos comunitarios y territoriales que tienen en cuenta la necesaria preservación de los lazos creados del sujeto (Galende, 1990). Nuestro país ha avanzado en procesos de reformas de este tipo, al principio desde políticas de alcance nacional y posteriormente con la descentralización de la gestión en materia de políticas sanitarias y de salud mental, el panorama se fue diversificando en cada provincia (Vezzeti, 1985). La provincia de Santa $\mathrm{Fe}$ ha tenido su propio movimiento de reformas, instaurando un proceso de transformación en las instituciones y las prácticas que han podido acumularse y reflejarse en la ley 
provincial $\mathrm{n}^{\circ} 10.772 / 91$ y su reglamentación (n²155/07) (Faraone et al., 2012).

Este movimiento se ha nutrido de los avances acaecidos en otros países, pero con el correr del tiempo adquirió una impronta propia marcada por las circunstancias y los actores locales. Uno de estos actores clave fue conocido como Movimiento de Trabajadores de Salud Mental, surgido en gran parte a partir de la experiencia Oliveros. Con este nombre se conocieron las iniciativas de un grupo de trabajadores de la colonia de Oliveros que, pasada la segunda mitad de la década del ' 90 , disputaron y ocuparon la dirección del establecimiento bajo un régimen colectivo conocido como Colegiado de Gestión. Desde este colegiado se impulsaron estrategias para implantar un proyecto de desmanicomialización.

De todas maneras, para poder diferenciarlo de las políticas de ajuste del período anterior que desembocaron en la deshospitalización y la desasistencia en nombre de la desmanicomialización, buscaron una nueva denominación que representase mejor el objetivo de la reforma que apuntaba a una deconstrucción del manicomio. Por ello denominaron a este proyecto como sustitución de lógicas manicomiales.

Un aspecto que caracterizó a la experiencia Oliveros fue el hecho de concebir su propia práctica desde una mirada clínico-política, al asumir que toda clínica y, en este caso, la clínica de la salud mental conlleva una lectura política. Esta clínica que llevaban adelante en el espacio de la colonia, se definía como una clínica ampliada que apuntaba a superar los límites biomédicos hegemónicos realizando un análisis de la vulnerabilidad.

Lo coyuntural de la experiencia Oliveros se fusionó con lo estructural de las condiciones políticas, sociales y económicas del país (Faraone y Valero, 2013). De esta manera, entre el 2001 y el 2003 se fue instituyendo este nuevo actor político en el ámbito de la provincia, más allá de la experiencia que fue su germen y que se denominó Movimiento de Trabajadores de la Salud Mental. Este movimiento se afirmó en la necesidad de sumar a las demandas sociales, el reclamo por la vulneración de derechos en los usuarios de salud mental y la necesidad de reformar el sistema de atención. Se fue conformando así un proceso instituyente cuyo objetivo central radicaba en la necesidad de inscribir en la agenda del estado provincial una política de salud mental, lo que implicaría necesariamente la recreación de la Dirección de Salud Mental -que había pasado a tener el rango de programa- hecho que se consumó en el 2004, constituyendo un acontecimiento. Otro suceso de importancia fue la reglamentación de la ley provincial de salud mental en el 2007, luego de un arduo trabajo que implicó serios debates entre los trabajadores respecto de la política de salud mental y la realidad de los equipos que se encontraban trabajando en los diferentes dispositivos de la dirección provincial. Aspectos importantes de esos planteos quedaron reflejados en varios artículos del reglamento, sobre todo en el 18, que prevé la transformación de los establecimientos de internación en vistas de la erradicación de las lógicas manicomiales, concibiéndola como "medicalización de los padecimientos subjetivos, internación por tiempo indeterminado, aislamiento social, silenciamiento, ausencia de estrategias terapéuticas complejas y singularizadas, perpetuación de las medidas de seguridad" (Decreto $\mathrm{n}^{\circ}$ 2155/07). Para ello, se prevé la promoción de la creación de servicios alternativos, incluyendo numerosos dispositivos y servicios de salud mental, entre los que se encuentran los servicios de salud mental en hospitales generales.

Del vasto panorama de dispositivos $y$ programas que surgieron de este proceso de sustitución de lógicas manicomiales, para este trabajo, nos abocaremos exclusivamente al hospital general como un escenario que permitirá focalizar en un aspecto asistencial de la práctica de internación por cuadros de crisis 
subjetivas.

Estas internaciones se venían dando en el marco de la ley de salud mental $n^{\circ} 10.772$ en los diferentes hospitales de la mano de los servicios de salud mental. Durante muchos años se insistía en que la declaración de esta ley no resolvía el problema, debido a que no contaba con una reglamentación que indicara los recursos que se deberían disponer para ello. El tema, al parecer, no tuvo muchas variaciones, sobre todo en lo que implicaba a los servicios de salud mental que las llevaban adelante, a pesar de lograrse la reglamentación del 2007. Las dificultades y los obstáculos persistían. Finalmente, el escenario del hospital volvió a adquirir una relevancia a partir del 2010 cuando nuevamente fue nombrado en la ley nacional $n^{\circ} 26.657$ como institución sustituta del manicomio para realizar una internación por crisis subjetivas. Esto revitalizó las discusiones y produjo diversas controversias que reflotaron la problematización de esta práctica de internación que venía dándose de alguna u otra manera en el hospital, más cerca de los márgenes institucionales.

Es evidente que las leyes son un avance para posibilitar prácticas innovadoras en salud mental y los procesos de reforma en nuestra provincia han podido demostrar su efectividad en la conformación de políticas públicas. Pero a pesar de esa evidencia, se sabe, la existencia de una ley no garantiza el funcionamiento de las instituciones acorde a los nuevos paradigmas en salud mental, que promueven su inclusión como un problema de salud pública. Para avanzar en un análisis más profundo de los obstáculos con los que se encuentra esta ley, es necesario problematizar acerca de los modos de producción de institución que surgen de las prácticas de los actores que se desenvuelven en ese terreno.

Por eso mismo, este trabajo se propone problematizar aspectos que emergen de las prácticas de internación en el hospital general, vinculados con los imaginarios sobre la locura en los trabajadores de la salud, y que actualizan una lógica manicomial, obstaculizando la realización de los nuevos dispositivos de tratamiento del padecimiento psíquico, presente en las leyes de salud mental y las políticas públicas.

\section{Imaginarios de la locura: el otro como peligroso.}

Por imaginario se entiende el registro que prevalece en la representación de una realidad, estableciendo un mecanismo esencialmente paranoico. Destacamos lo imaginario en relación con las identificaciones formadoras del sujeto (yo) y sus relaciones con lo real, de carácter ilusorio (Lacan, 1996). Lo imaginario funciona como señuelo que obtura lo hueco, lo que falta, en una completud aparente e ilusoria de la imagen (júbilo) que cuando falla desencadena la agresividad en el rechazo. El rechazo es absoluto porque hay una amenaza a nivel de la propia imagen.

Entonces, en el imaginario de la locura intervienen los modos tradicionales de pensarla y abordarla, insisten las representaciones que dieron origen al asilo, el saber psiquiátrico y la disciplina criminológica, encargados de la investigación de las anormalidades del instinto, su actividad mórbida asociada al crimen, su origen enigmático, puesto que el instinto representaba un fondo ignorado del que podían surgir anormalidades desconocidas y por lo tanto peligrosas para el orden público (Galende, 1997).

En estos imaginarios insisten, como resto del trauma del primer encuentro, las ideas de degeneración y peligrosidad en un estado de latencia que, parece, ha sobrevivido a los posteriores intentos de reformas (Zaffaroni, 2011). Queda como resto la asociación del tratamiento a un castigo, puesto que el que no comprendía la criminalidad de su acto no podía ser culpado de un delito pero en lugar del castigo de la pena, tendrá lugar el encie- 
rro manicomial. "El bajo oficio de castigar se convierte así en el hermoso oficio de curar" (Foucault, 2007a: 35). Psiquiatrización de la vida familiar, de la infancia, en busca del cúmulo instintivo morboso, desconocido y peligroso para el orden social en donde emerge la figura del anormal, del individuo incorregible, expresada en sus distintas manifestaciones (el monstruo, el masturbador, y el indócil) y que justifica el accionar disciplinario de la sociedad en manos de las instituciones correctoras (Foucault, 2007b).

El poder de normalización produce enfermedad mental, duplica cruelmente el padecimiento como objeto del cual servirse para legitimar su posición de poder-saber desde donde se implementará todo el funcionamiento disciplinario (Foucault, 2009). Es el lugar en donde se desarrolla una técnica positiva de intervención directa sobre el cuerpo que busca gobernar al anormal desde este despliegue disciplinario, "anormal" que se construye a partir de estos agregados al padecimiento que, en calidad de maltrato, forman la enfermedad institucional propia de las instituciones totales (Goffman, 1961).

En su pretensión terapéutica, el asilo rompe con los lazos familiares y de la comunidad con una intención higiénica, al asumir que es en ese contexto que el individuo se enferma. Si la institución familiar no funcionó como instancia disciplinaria, el individuo es captado por la institución asilar para corregir en él lo que ha escapado al poder de normalización. En este sentido se lo concibe como un niño a civilizar y educar en los valores de la realidad, al modo de una ortopedia moral y psicológica que sustituye la función de la familia (Foucault, 1998).

De todas formas, el planteo del imaginario que despierta la locura y las figuras que convoca, permiten pensar hoy en día en el tipo de respuestas que genera, pudiendo ir desde este enfoque disciplinario y normalizador de un niño o un monstruo para el cual se acude al poder de normalización (representado en el manicomio), hasta el extremo de la desasistencia por la falta de instituciones acordes y el rechazo de las instituciones de salud.

Las posibilidades de pensar el dispositivo del manicomio y sus modos característicos es fruto de las reformas, puesto que uno de los puntos más destacados es diferenciar la institución manicomial surgida en el siglo XVIII y que llega, con algunas variaciones, hasta nuestros días; de lo manicomial como institución, en relación a los modos de trabajo y a las prácticas que se desarrollan, que no tienen que ver directamente con una institución particular del tipo asilo u hospital monovalente, sino que funcionan como una lógica manicomial que opera extramuros (Rotelli et al., 1987). Ha sido una advertencia de los teóricos a nivel mundial: el hospital psiquiátrico no deja de funcionar sólo con su cierre y prohibición; y es en esta distinción que se fundamenta el presente trabajo para avanzar sobre las particularidades que suceden en el trabajo cotidiano de la institución, más allá de la legislación vigente, que posibiliten o no otro tipo de abordajes en salud mental.

En el mismo despliegue de esta lógica manicomial se advierte el no reconocimiento del otro como semejante. Ese aspecto, propio de la crueldad, establece un camino que difícilmente se corra de la expulsión, el rechazo, la agresividad, encausados por los imaginarios que se despiertan con la figura del loco como ajeno, externo absoluto, depositario de las estigmatizaciones.

Suele resultar muy difícil romper con el discurso hegemónico de la medicina clásica, cuando se instaura como discurso amo. Allí, el acto del diagnóstico es un acto de dominio (Clavreul, 1985), no ya de la persona del médico hacia la persona del paciente, sino desde un saber totalizador con que se lo mira, puesto que el médico queda despojado en cuanto persona, oficiando de funcionario de un saber absoluto que lo excede y del cual es un portavoz. Como 
efecto, el paciente también queda despojado del propio padecimiento de su cuerpo puesto que ya no garantiza su propia coherencia, se ha alejado de su normatividad. El médico será representante e intérprete del saber ante el enfermo y su coherencia es proporcionada por la cientificidad de la permanencia.

Entonces, en relación a una verdadera transformación de los servicios asistenciales, se destaca que el sostenimiento del manicomio va más allá de su cierre, reforma o adecuación. Por ello, al hablar de lo manicomial como lógica emergente en las prácticas, a lo que se hace referencia es a tres instancias que deben estar presentes para su persistencia: el juicio de peligrosidad, la necesidad de control y vigilancia de lo anormal y la idea de irreversibilidad y cronicidad del padecimiento psíquico (Barraco, 2006).

\section{El loco en el hospital.}

Partimos de la hipótesis de que un hospital general puede ser a veces una institución total que impide el trabajo con el inconsciente, repitiendo las lógicas propias de la institución manicomial, incluso a pesar de la legalidad que, a partir de las nuevas leyes, hacen de marco a las prácticas. Interesa reflejar aquí cómo el ámbito del hospital general forma parte de un escenario en donde podrá notarse el juego de los distintos elementos, dispares y no siempre explicitados, que en relación a la locura, constituyen un imaginario social presente en los modos de abordaje de los trabajadores de la salud y que podrían reflejarse en los pacientes al modo de una estigmatización, instaurando la lógica manicomial que obstaculiza los procesos de atención pretendidos desde las políticas públicas

En el hospital, el dispositivo que se ha instituido para trabajar sobre estos aspectos es la interconsulta, un analizador primordial del tipo de institución que se establece, de las posibilidades de alojar a un sujeto. En tanto dispositivo, ha tenido sus variaciones de sentido. Hoy podemos hablar de la interconsulta como un dispositivo que permite la introducción de otro discurso en el

trabajo del hospital. Para esto el psicoanálisis es una herramienta valiosa, que nos permite escuchar los efectos $y$, desde su ética, propiciar la emergencia subjetiva allí donde el sujeto suele quedar convertido en objeto. En este sentido, el dispositivo de la interconsulta constituye un encuentro necesario para apostar a la construcción de una clínica que se base en el caso por caso y posibilite las acumulaciones instituyentes de nuevos procesos de atención-cuidado para los pacientes internados con algún tipo de padecimiento psíquico.

Ahora bien, este dispositivo no conlleva un sentido unívoco de trabajo clínico. Por el contrario, produce diferentes tipos de institución. Fueron las vicisitudes de la relación médico-paciente las que le dieron origen, con el fin de intervenir sobre los conflictos para aliviar la crisis en la relación y liberar las acciones hacia las metas pre-supuestas. En sus orígenes, apuntaba a completar la educación médica para devolver al médico su máximo potencial terapéutico (Ferrari et al., 1971). Esta versión coincide con una concepción funcionalista de la institución, es decir, que se parte de aceptar que ha sido creada con determinados fines -que tienen que ver con la salud-y que en el transcurso algo puede desviarse, alterar su buen funcionamiento y originar un conflicto, no siempre explicitado. La interconsulta estaría allí a la hora de volver a encausar, ajustar, conducir a la institución hospitalaria por sus carriles adecuados de funcionamiento.

Ahora bien, las interconsultas que suelen llegar al servicio de Psicología se dirigen a problemas que parecen surgir, para los médicos, al margen de su trabajo y pueden, por lo tanto, constituir un enigma o ser rechazados, de diferentes formas, por considerarlas accesorias a la clínica biomédica (Grande, 
s/f). Muchas veces lo accesorio es lo que se termina expulsando, actuando o rechazando. El hecho de que se convierte en enigma posibilita que lo que está en el margen pueda ser interrogado por quienes realizan una práctica y se sientan interpelados por eso que los atraviesa. Esto resulta una apuesta renovable en cada caso, con las dificultades propias de una sala de internación en la que prima el discurso médico tendiente a dejar por fuera las singularidades propias de la economía libidinal del sujeto que padece con su cuerpo y no solo con su organismo.

Por ello, analizar las respuestas que la institución ofrece a los emergentes de un padecimiento psíquico permite vislumbrar qué del manicomio retorna en las prácticas, así como las posibilidades de producir otro orden de cuidados. Sostenemos que la actuación de estos imaginarios por sí solos coagulan las respuestas antes de lanzar una pregunta por el padecimiento, su razón de ser, su pronóstico. Dificultan que el sujeto que padece sea visto como paciente. Los tiempos del hospital suelen abonar a ello. Allí las respuestas institucionales emergen entre las que ubican al manicomio como único lugar posible, y las que rechazan lo que consideran accesorio, desasistiendo. Esto va en detrimento de la construcción de una clínica de salud mental apropiada que incluya al cuidado atento y pertinente como forma de la abstinencia (Ulloa, 2011).

Este escrito resulta de un análisis bibliográfico del problema que permitió la identificación de ideas y preguntas clave que facilitaron, luego, llevar adelante entrevistas semi-estructuradas a informantes clave del trabajo diario en el hospital (enfermeros, médica, psicóloga). En ellas se puntualizó sobre los aspectos centrales que hacen a la práctica de internación, elaborados a partir de la propia experiencia de trabajo en el servicio de Psicología mediante el dispositivo de la interconsulta entre el 2010 y el 2013. Por otra par- te, se realizó una entrevista a un representante de la gestión provincial y una observación no participante en las capacitaciones a enfermeros de hospitales generales llevadas a cabo por la Dirección Provincial de Salud Mental.

\section{Algunos elementos que resultaron de este trabajo.}

Como elementos destacados del análisis bibliográfico de los procesos de reformas del manicomio (Foucault, 1992, 1998, 2007, 2009; Galende, 1997; Basaglia, 2008, 2009; Cooper, 1976; Zsasz, 1994), podemos precisar que uno de los aspectos sobresalientes para lograr avances en los nuevos dispositivos de abordaje, sobre todo en el caso italiano, está relacionado con la importancia de modificar la representación que se tiene de la locura por parte de la sociedad. La importancia de ello radica en que la sociedad, al haber asimilado los conceptos de las disciplinas que históricamente han intervenido en el tema, reclaman su actuación cuando la locura acecha como peligro. Este punto es interesante porque nos lleva a pensar en la necesidad de atravesar un proceso de deconstrucción de los saberes disciplinarios que operan a contra mano de las reformas, repitiendo un esquema de representación en el cual la locura aparece como enigma a develar por la psiquiatría y como peligro a encerrar en el manicomio. Esta deconstrucción fue la que se intentó llevar adelante desde la experiencia Oliveros.

De la mano de esto, otro aspecto relevado se refiere a la relación existente entre la enfermedad mental y la exclusión social. Esto implica que toda reforma, necesariamente, debe asumir una postura de crítica radical, de rechazo de la exclusión, de subversión, no ya del manicomio en sí, sino de los discursos que sostienen la crueldad. En la experiencia italiana se advierte aquello que podemos también reconocer en nuestro medio: los enfermos internados pertenecen al sector de las clases 
sociales oprimidas y el hospital psiquiátrico es un medio de control social (Basaglia, 2008). De ahí que la ley 26.657 establezca los medios necesarios para resguardar los derechos vulnerados de los usuarios y exprese que la salud mental se asocia a la defensa y consecución de los derechos humanos.

Según el recorrido histórico de las reformas de la atención, la vulneración de derechos humanos que implica el manicomio pudo ser vislumbrado y denunciado al derribar los muros del gran encierro (Foucault, 1998). Esto significó que comiencen a surgir directivas y normas que regulaban a las instituciones y los profesionales, para prevenir tal vulneración y resguardar los derechos de los pacientes, incluyéndose posteriormente en los lineamientos de salud transnacionales de los organismos internacionales (OMS/OPS). Ahora bien, el hecho de haber sido descubierta esta vulneración no garantiza que en el devenir de la historia no existan puntos de renegación, en donde estos aspectos esenciales y definitorios de las prácticas como el respeto por los derechos humanos, sean objeto de olvido, y lo ya rechazado pase a ser legitimado nuevamente. ${ }^{3}$

Estas legitimaciones se perciben, al menos, en dos planos distintos. Uno es el plano de las políticas y otro el de las prácticas concretas, más allá de que necesariamente estén relacionados. Este trabajo se focaliza en el nivel de las prácticas. De todas maneras, un análisis que ubique su eje en las políticas de salud mental debería incluir los aspectos ideológicos que las sostienen, a pesar de que en numerosas ocasiones se presentan camufla-

\footnotetext{
${ }^{3}$ Basta advertir hoy en día las nuevas políticas nacionales que parten desde gobierno nacional y sus embestidas a la ley 26.657, en franco retroceso del proceso impulsado a partir de 2010. Entre las modificaciones establecidas se aprecia un claro beneficio hacia la corporación psiquiátrica y farmacológica, en detrimento de las perspectivas interdisciplinarias y comunitarias que resguardan a lo usuarios.
}

dos con conceptos extraídos de paradigmas opuestos.

De todos modos, lo que interesa resaltar es que, más allá de las políticas y los límites impuestos por el muro de la institución, el manicomio puede introducirse por los vértices imaginarios de los trabajadores y profesionales de la salud, produciendo modalidades de prácticas que perpetúan la institución manicomial. Esta distinción permite interrogarse por los fundamentos de las propuestas de desinstitucionalización, más allá de la figura, también imaginaria, del cierre de los manicomios.

\section{Reforma local: la sustitución de lógicas manicomiales.}

Sobre el análisis de la reforma local, (Gerlero y Ausburguer, 2012; Faraone y Valero, 2013) sintetizamos que las transformaciones de las prácticas de salud mental en la provincia de Santa Fe se fueron dando en el marco de las transformaciones de las prácticas de salud en general, en vistas a conformar una red de salud en donde el proceso de atención contemple la salud como un derecho. El proceso de sustitución de lógicas manicomiales llevado adelante fue configurando un panorama particular de dispositivos de salud mental tal como se encuentran desplegados hoy en día. La particularidad consiste en que estas transformaciones se fueron produciendo de una manera heterogénea e incluso muchas veces al margen de la legalidad existente, encontrando un nuevo horizonte a partir de la Ley Nacional de Salud Mental.

Respecto a la institucionalidad para hacer frente a la demanda asistencial, a lo largo del territorio provincial conviven, junto con los hospitales psiquiátricos, diferentes dispositivos que intentan abordar la problemática de la salud mental con una lógica que recupera los avances de estas transformaciones. Queda conformado así un complejo campo que con- 
tinúa atravesando modificaciones, a partir de los cambios de gestión y de las diversas problemáticas que afronta (Herrman, 2007).

En lo que refiere a las internaciones en el hospital general como lugar sustituto, hoy en día son más habituales. La existencia de la ley marca una legalidad que lleva a que se incluyan en el devenir cotidiano del hospital, aunque de manera heterogénea. En este escenario diverso, en el que conviven distintas prácticas de salud, se percibe la insistencia de algunos profesionales en las lógicas tradicionales que tienen al hospital psiquiátrico como eje de sus intervenciones. Al presentarse la problemática de salud mental, suele entrar directamente en el circuito de la derivación, aduciendo la necesidad de contar con una mayor complejidad del sistema de salud. Esto es favorecido por el hecho de que los hospitales psiquiátricos aún siguen funcionando en la red como hospitales monovalentes. También se refleja la dificultad de la articulación dentro del sistema de salud, impidiendo de esta forma la evaluación del modo de operación de la estigmatización del diagnóstico cuando es leído desde la clínica psiquiátrica. En este sentido, la estigmatización obedece a la siguiente afirmación: "Cuando la locura habla, se enfrenta con la institución de la locura" (Mannoni, 1983:51). Vemos entonces cómo el criterio de internación puede ubicarse no ya desde una estrategia clínica sino desde un criterio ideológico y masivo que contrapone hospital y manicomio en función de la demanda social.

Además, quedan en evidencia las limitaciones institucionales para trabajar con el padecimiento psíquico. Estas limitaciones son propias de una institución que basa su origen en el modelo médico hegemónico, para el cual no cabe la pregunta por el padecimiento psíquico, sino sólo cuando remite a alguna alteración orgánica o existe una fuerte asociación (como en los casos de pasajes al acto con lesiones importantes en el cuerpo). En estos casos, la pregunta busca resolverse en la derivación al psiquiatra como médico especialista. Es por esta razón que muchas veces la dolencia física de un sujeto, si la hubiere, sirve como excusa para efectivizar una internación y así abordar la crisis subjetiva. Pero también, esta primacía de lo orgánico, es la que subyace cuando se considera que el período agudo de esta dolencia ha finalizado, y suceden las altas sorpresivas de un paciente internado. El ingreso y el alta de un paciente están fuertemente condicionados a la presencia y al desarrollo de los indicadores orgánicos del padecimiento. Las limitaciones institucionales operan pretendiendo hacer coincidir lo orgánico y lo subjetivo en el proceso de internación, por más que impliquen tiempos muy diferentes. Quienes se ubican como voceros de estas limitaciones aducen que, por sus características, el hospital no sería terapéutico, pero en pocos casos aparece la pregunta que permita construir una estrategia terapéutica para alguien que ingreso - de hecho- a la institución.

El trabajo con el padecimiento psíquico implica, como condición general, poner en cuestión el estatuto que puede tener una internación para un sujeto en crisis, y eso debe hacerse en cada una de las situaciones que llegan al hospital. Si tenemos en cuenta que ésos pueden ser momentos de ruptura de las articulaciones simbólicas que posibilitan a un sujeto el armado de su cotidiano estar en el mundo (Fermoso, 1997) -cayéndose fuera de la escena y del discurso- la internación bien puede constituir la intervención de una temporalidad necesaria para lograr un anudamiento subjetivo. Esta práctica podría ser, entonces, una vía para lograr la restitución de su capacidad para armar la escena del mundo y poder transitarla desde el lazo social.

En lugar de ello, se destacan las dificultades y obstáculos en el proceso de atención de un paciente que ingresa con una crisis subjetiva o un diagnóstico psiquiátrico. En un primer plano, aparecen las complicaciones 
que presenta el hospital general para desempeñarse como alternativa al manicomio. Una de las que se presenta con mayor énfasis es la falta de adecuación estructural de la institución para alojar este tipo de pacientes, aunque se diferencia entre lo que serían pacientes deprimidos, apáticos o introvertidos de aquellos con un cuadro de psicosis o melancolía. Esta limitación estructural originaría una imposibilidad de brindar ciertos cuidados, ya que el hospital no garantiza la permanencia del paciente ni su vigilancia permanente. Es decir, que respecto de la atención o al cuidado de estos pacientes, lo que surge es la necesidad de tutela y vigilancia. De esta manera, no parece diferenciarse de lo que constituye el dispositivo de control manicomial propiamente dicho. Esta función institucional reclamada es la que proviene de la demanda social respecto de la locura, aquella que pide custodiar, ordenar, encausar y volver a la razón lo que se presenta como anormal o desviado en el campo social.

Podemos decir que no se piensa a la internación como la posibilidad de brindar un cuidado, que, en términos subjetivos, puede permitir que la institución funcione como una instancia necesaria en un momento de crisis subjetiva, de suspensión de las articulaciones simbólicas de un sujeto, en vistas a posibilitar la restitución por vía de alguna metáfora alternativa.

Lo que emerge como práctica ante esta limitación retorna del manicomio, sea vía la medicalización compulsiva o los acompañamientos terapéuticos de veinticuatro horas tendientes a garantizar un control y vigilancia. Queda en un margen la posibilidad de pensar los criterios para una medicación que no implique sólo una sedación, así como la pertinencia o no de un acompañamiento terapéutico. En los acompañamientos de veinticuatro horas y durante el tiempo que lleve la internación como condición es en donde puede leerse una totalización de la institución. Es decir, que el hospital general toma una va- riante de las instituciones totales (Goffman, 2009). En esos modos de funcionamiento la cuestión de salud mental no queda incluida en la polivalencia que llega al hospital, y por tanto, resulta un agregado para el trabajo -sobre todo de enfermería- y por tanto, una carga y un desborde.

Diferente parece ser otro aspecto que se reclama fuertemente relacionado con los lugares intermedios, instituciones alternativas, que ayuden a completar el proceso de atención que se inicia desde el hospital. Sobre todo, cuando se refieren a tratamientos de adicciones, pero en general a los tratamientos de salud mental, en los que a veces se necesita de algún lugar previo hasta que estén dadas las condiciones para que el paciente regrese a su casa.

Un elemento interesante que aparece fuertemente en el servicio de enfermería es el reclamo por capacitaciones para alojar una crisis subjetiva, asociada con conductas agresivas y peligrosas para la integridad física de los trabajadores. La capacitación estaría fundamentada en una formación específica para atender a estos pacientes o contener una crisis, es decir, una especialidad de la profesión que pone en falta a aquellos que no la poseen. ¿Qué tipo de capacitación teórica o práctica puede resolver el obstáculo de los imaginarios? La pregunta tiene un valor retórico en el sentido en que intentamos desdoblar nuestro problema en los términos de lo que es pasible de acumularse como saber positivo, especificidad de una disciplina, de lo que emerge en los vértices de la subjetividad, y, por ende, de las instituciones.

El pedido de capacitaciones de los trabajadores parece fundamentarse, también, en cierta soledad a la hora de responder a esta demanda para la cual no fueron consultados. Los servicios de Psicología y Psiquiatría no cuentan con modalidad de guardia pasiva y por tanto se puede recurrir a ellos sólo por la mañana. Es decir, que los trabajadores del 
hospital que no pertenecen a estos servicios, deben actuar solos ante estos pacientes (sic) y deben hacerlo sólo por la existencia de una ley que así lo indica.

En la nominación de estos pacientes que hacen algunos trabajadores -alineadas al significante de paciente psiquiátrico-, se evidencia también un discurso que los objetaliza. En esa objetalización intervienen de modo privilegiado los imaginarios construidos. Esta modalidad da por sentado que las conductas a seguir en la atención serían las mismas en cada uno de esos casos, dejando por fuera la clínica de lo singular. La objetalización del padecimiento, la construcción de la entidad enfermedad mental o crisis subjetiva lleva a reclamar cierta especificidad en la práctica, o bien un saber que detenta otro y no un aspecto propio de la subjetividad del que padece susceptible de ser escuchada a través de la puesta en juego de una particular sensibilidad, a veces presente en la simplicidad del sentido común. Es decir, hay un límite que, para el propio trabajo, lo constituye la suposición de que hay otro que sabe qué hacer con la locura.

Estos aspectos alejan las posibilidades de pensar una clínica ampliada (Sousa Campos, 1996) en el hospital, clínica que repara en sujetos para los cuales el enfermar incluye los aspectos subjetivos del que padece, sin objetalizar la enfermedad en una entidad aislada, como enfermedad orgánica o bien esos aspectos subjetivos como enfermedad mental. Esta división continúa apareciendo en los discursos de médicos y enfermeros y en esa medida se transfiere a las prácticas circunscribiendo las posibilidades de ampliación de la clínica a la singularidad de los profesionales tratantes.

El trabajo clínico en salud mental en el hospital, con estas particularidades que venimos enunciando, originan importantes malestares al interior de los servicios. Se producen repliegues defensivos en los trabajadores que hacen más dificultoso el abordaje. Cuando el padecimiento psíquico se presenta en los pacientes, se lo concibe como un agregado, un peso más, un motivo de desborde sin razón que empobrece el quehacer clínico, la propia tarea. Este juego muchas veces termina en una encerrona en el que los marcos de referencia se achican y oprimen dando lugar a un escenario de imágenes en el que quedan cautivadas las posiciones de víctimas y victimarios. La agresividad imaginaria que emerge tensa allí hacia la expulsión.

La fuerte dependencia a la singularidad de los profesionales tratantes en lo que refiere a las intenciones de propiciar una clínica ampliada adquiere relevancia si tenemos en cuenta que el trabajador de la salud es un trabajador del conocimiento y del lenguaje (Spinelli, 2010). Entonces, en su proceso de trabajo se ponen en juego no sólo sus saberes sino cuestiones subjetivas tales como sus valores, sus ideologías, que se suman a los imaginarios que despierta la locura respecto de su peligrosidad, su agresividad o su violencia innata, su anormalidad, su irresponsabilidad, y su entidad objetivable. Esta autonomía (Spinelli, 2010) del trabajador de la salud facilita así un terreno para las diferentes formas de tratar, que incluyen tanto el destrato o maltrato, con sus variantes de expulsión de lo que se concibe como ajeno, hasta las formas más genuinas de cuidado al semejante.

De la mano de la objetalización de los pacientes y del pedido de capacitaciones se asocia un tercer elemento emergente: el pedido de confección de protocolos institucionales que otorguen un ordenamiento a los diferentes actores. Lo interesante aquí sería preguntarse por la funcionalidad de un protocolo de tratamiento para estas situaciones en la medida en que se puede ubicar como un pedido de homogeneizar la problemática desde la perspectiva de la medicina biologicista que tiene sus métodos y procedimientos rigurosos. Pero sobre todo es necesario advertir que la tendencia a la burocratización de las prácticas 
en salud mental procura un imposible: el borramiento del conflicto. Esta burocratización puede constituir un mandato de la institución cuando se la piensa desde la perspectiva funcionalista, en la que el ideal pretende decir cómo se hace, algo que supuestamente tranquiliza pero que desoye lo propio de las prácticas, sus obstáculos y dificultades inherentes, así como el hallazgo de lo distinto y lo nuevo emergente.

Para poder acercarse más a una clínica que contemple el padecimiento psíquico de los pacientes en el hospital general, y pueda proponer formas innovadoras en el proceso de atención, la discusión continua de los actores intervinientes resulta una condición de posibilidad. Sobre todo, cuando la institución intenta silenciar los interrogantes en esos procedimientos acabados y homogeneizados. Pero también, esta discusión se torna necesaria cuando aparecen los obstáculos inherentes, las formas cristalizadas de dar tratamiento, en vistas a promover que la labor se encamine a la construcción de un pronóstico y un para qué prospectivo (Ulloa, 2011) que puedan producir de un sujeto, un paciente. Esta propuesta responde al cómo hacer con el ir haciendo, a la burocratización de la práctica, con el intento de recuperar en ella la subjetividad del que padece, incluyendo los obstáculos estructurales pretendidamente desoídos por el ideal de la institución y convertidos en impedimento (Baños, 2012).

\section{Algunas ideas finales.}

Para finalizar, se puntualizarán algunas ideas que han aproximado una comprensión del problema, reflexionando en torno a la hipótesis que lo recorre y al objetivo propuesto. En ese sentido retomamos del inicio la propuesta de problematizar la práctica de internación en el hospital general como práctica que, más allá de su previsión en la ley de salud mental y las políticas que la promueven, se desarrolla de manera diversa, pudiendo actualizar una lógica manicomial al interior de la institución hospital general.

Podemos ver cómo el llamado paciente de salud mental, con los imaginarios que despierta en los trabajadores, corre con mayores dificultades a la hora de constituirse como tal en estos espacios sustitutivos en que aparece ajeno a la polivalencia. Insiste la dificultad de la inclusión de la salud mental en un ámbito de la salud. Este sentimiento de ajenidad va más allá de una especificidad disciplinaria y de una adecuación estructural de la institución que, si bien son consideradas necesarias, no garantizan las formas de tratamiento previstas por las leyes de salud mental.

Así como decimos que, en todo proceso de enfermedad, tanto en su constitución como en su forma expresiva, la cuestión subjetiva es ineludible, también esto se advierte en aquellos encargados del proceso de atención. Más allá de un saber faltante, muchas veces se trata de una posición subjetiva que se defiende de lo extraño que la locura despierta, de la confrontación con el vacío en que el otro se mueve. Aquello que queda rechazado en nosotros y el discurso de la locura presentifica: la muerte, el sexo, la libertad; aquello que en las neurosis forma parte del retorno de lo reprimido al modo del enigma a descifrar. El saber, ya sea asumido por unos o proyectado en otros, se ubica como un modo de obturar esta confrontación y no garantiza que el loco deje de presentarse como lo extraño y no como un semejante.

La preparación para recibir a estos pacientes no pasa sólo por un contenido teórico que se agregue, que haga el juego a la falta, sino que necesariamente se debería actuar sobre los imaginarios presentes, descompletar el efecto totalizador, de completud aparente, propio de las peripecias imaginarias de los escenarios especulares. Hay ciertos aspectos de las prácticas con la locura, con el padecer subjetivo, que forman parte del 
sentido común sobre el cuidado del semejante, y eso no excede a las prácticas de un hospital general.

Sin embargo, esto no exime la especificidad de las disciplinas psi a la hora de llevar adelante estos procesos. Vendrá desde estas disciplinas el aporte para conceptualizar el estatuto que puede tener una internación, el abordaje del síntoma, las implicancias subjetivas de una crisis, lo terapéutico que podría ser un paso por el hospital general, en el marco de un tratamiento. Pero también, y Basaglia refería claramente este asunto, "se trata de violentar a la sociedad desde el accionar de un movimiento y a la vez estar presentes allí como clínicos para hacerse cargo de esas acciones y ayudar a entender a la comunidad de qué se trata la presencia de una persona loca en la sociedad" (Basaglia, 2008:36).

Por ello, los avatares en la implementación de la ley, las posibilidades y obstáculos que hacen a su viabilidad, no corresponden únicamente a las cuestiones técnicas que instaura sino también a cuestiones ideológicas y políticas. Es decir, que la viabilidad es algo que debe construirse puesto que está relacionada a la confluencia de los diferentes intereses (económicos, corporativos, personales, etc.) de los actores que están en juego, teniendo en cuenta que, si bien pueden encaminarse hacia un cuestionamiento y reformulación de las prácticas, la misma no se plasma de manera sustancial ni sostenida en el tiempo.

En este sentido, puesto que la ley apunta a cerrar el manicomio, podríamos decir que ninguna de las formas que adquiere el manicomio podrán cerrarse simbólicamente hasta tanto aquellos que lo sostienen queden advertidos, no sólo de su ineficacia terapéutica sino de la innecesariedad para la práctica que sostienen. Pero para que esto pueda suceder es necesario también demostrar cómo los modelos alternativos sí conllevan un potencial terapéutico para quienes sufren algún padecimiento psíquico. Recién ahí, el manicomio -y las formas de crueldad que implica- podrá considerarse realmente obsoleto.

\section{Referencias:}

- BAÑOS, L. Y STEINBERG, I. (2012). Dificultades de la práctica del psicoanálisis. Rosario: Homo Sapiens.

- BASAgLiA, F. (2008). La condena de ser loco y pobre. Alternativas al manicomio. Buenos Aires: Topia.

- (1970) La institución negada Informe de un Hospital Psiquiátrico. Barcelona: Barral.

- (1977) La mayoría marginada. La ideología del control social. Barcelona: Laia.

- BARRACO, A.. https://www.topia.com. ar/articulos/demanicomializaci\% $\quad$ C3\% B3n-los-1\%C3\%ADmites-de-la-ley

- COOPER, D. (1976). Psiquiatría y antipsiquiatría. Buenos Aires: Locus Hypocampus.

- Clavreul, J. (1985). El orden médico. Buenos Aires: Argot.

- FARAONE, S Y VALERO, A. (COMP) (2013). Dilemas en Salud Mental. Sustitución de las lógicas manicomiales. Buenos Aires: Madres de Plaza de Mayo.

- FARAONE, S.; VALERO, A.; TORRICELLI, F; ROSENDO, E; MÉNDEZ, M. Y GELLER, Y. (2012). "Accesibilidad y derechos humanos. Análisis de los procesos de atención alternativos al modelo asilar en Santa Fe y Tierra del Fuego". Revista Argentina de Salud Pública Vol. 3. n 12.

- FERMOSO, J. L. (1997). "La internación: un dispositivo que tiene su lógica”. En Psicoanálisis y el Hospital. $\mathrm{N}^{\circ} 11$

- FERRARI, H.; LUCHINA, I. Y LUCHINA, N. (1971). La interconsulta médico-psicológica en el marco hospitalario. Buenos Aires: Nueva Visión.

- FOUCAUlT, M. (1998). Historia de la locura en la época clásica. Bogotá: Fondo de Cultura Económica.

- -(2007a) Los anormales. Buenos Aires: 
Fondo de Cultura Económica

- -(2007b) El poder psiquiátrico. Buenos Aires: Fondo de Cultura Económica

- -(2009) Vigilar y castigar. Buenos Aires: Siglo XXI

- GALENDE, E. (1997). De un horizonte incierto. Psicoanálisis y salud mental en la sociedad actual. Buenos Aires: Paidos

- -(1990) Psicoanálisis y Salud Mental. Buenos Aires: Paidos.

- gerlero, S. AUSBUrguer C. (COMP.) (2012). Salud Mental en Argentina: Avances, Tensiones y Desafíos. Rosario: Laborde.

- GOFFMAN E. (2009). Internados. Buenos Aires: Amorrortu.

- GRANDE, S. (S/F) Interconsulta. Material interno del seminario de pregrado de la cátedra de Residencia Cínica "Del riesgo a la vulnerabilidad". Inédito.

- HERrMAN, J (COMP.) (2007). Memorias de la Capacitación. Gente Necesaria para construir una historia: Proyecto troncal de capacitación. Proyecto troncal de capacitación 2006 2007. Santa Fe: Ministerio de Salud de la Provincia de Santa Fe.

- LACAN, J. (1996). Los escritos técnicos de Freud. Buenos Aires: Paidós

- MANNONI, M. (1983). El psiquiatra, su loco y el psicoanálisis. México: Siglo XXI

- ROTELLI, F; DE LEONARDIS, O Y MAURI, D. (1987). "Desinstitucionalización: otra vía (la reforma psiquiátrica italiana en el contexto de la Europa Occidentaly de los paises avanzados”. En Revista Asociación Española de Neuropsiquiatría Vol. VII n ${ }^{\circ} 21$

- SOUZA CAMPOS, G (1996). La clínica del sujeto. Por una clínica reformulada y ampliada. Disponible en http://www.salud. rionegro.go v.ar/bibliot eca/docu mentos/salud_mental/Gestion $\% 20$ en $\% 20$ Salud. $\% 20$ Sousa \%20Campos.pdf

- SPINELLI, H. (2010). "La dimensión del campo de la salud en la Argentina”. En Salud Colectiva. Buenos Aires Vol. 6 n 3.

- SZASZ, T. (1994). El mito de la enfermedad mental. Buenos Aires: Amorrortu.

- Ulloa, F. (2011) Salud ele-mental. Con toda la mar detrás. Buenos Aires: del Zorzal

- VEZZETTI, H. (1985). La locura en la Argentina. Buenos Aires: Paidos.

- ZAFFARONI, R. (2011). "Criminología y Psiquiatría: El trauma del primer encuentro". En Salud Mental y Comunidad. Año $1 \mathrm{n}^{\circ} 1$

\section{Legislación:}

- Ley Nacional de Salud Mental No 26.657 Congreso Argentino 25 de noviembre de 2010. Promulgada por la presidenta de la nación, Dra Cristina Fernández de Kirchner, el 2 de diciembre de 2010. Publicada en el Boletín Oficial Nº 32041 el 3 de diciembre de 2010.

- Ley Provincial de Salud Mental No 10.772. Promulgada por la legislatura de la provincia de Santa Fe el 26 de diciembre de 1991. Publicada en el Boletín Oficial del día 11 de febrero de 1992.

- Decreto Reglamentario $N^{\circ}$ 2155/2007 de la Ley Provicial de Salud Mental $N^{\circ}$ 10.772. 21 de septiembre de 2007.

- Decreto Reglamentario n 603/2013 de la Ley Nacional de Salud Mental N²6.657 reglamentado por la presidenta de la nación, Dra Cristina Fernández de Kirchner, el 28 de mayo de 2013. Publicada en el Boletín Oficial No 32649 el 29 de mayo de 2013. 